\title{
Mortality associated with odontogenic infection!
}

\author{
A. W. Green, ' E. A. Flower, ${ }^{2}$ and N. E. New, ${ }^{3}$
}

\section{Odontogenic causes are the most common source for spreading maxillo-facial infections. ' These infections can develop into life threatening events. However a fatal outcome is fortunately rare and is generally associated with an immunocompromised status. ${ }^{2,3}$ This case report highlights a spreading maxillo-facial infection, which resulted in massive haemorrhage from the subclavian vein into the pleural cavity and subsequent death of a young fit male patient.}

\begin{abstract}
25-year-old male was referred with Arapidly advancing bilateral neck swelling of 3 days duration. Examination revealed marked trismus (opening limited to approximately $1 \mathrm{~cm}$ ), raised tongue and slight dysphagia but no respiratory problems.

The patient admitted to being an irregular dental attender, with a history of intermittent dental pain over the previous 4 months. A panoramic radiograph showed heavily carious teeth in both the right and left lower quadrants with associated periapical pathology (Fig. 1). There was no relevant previous medical history.

The patient was taken to theatre, within 3 hours of presentation, for removal of the carious teeth and extra-oral drainage.

A fibre-optic awake intubation was performed and intra-venous antibiotics started at induction $(500 \mathrm{mg}$ amoxycillin and $500 \mathrm{mg}$ metronidazole both three times a day). Extra-oral incisions were performed and the carious teeth were extracted. No frank pus was located but extra-oral drains were placed. Over the next 2 days his condition significantly improved and he was discharged home.
\end{abstract}

${ }^{1}$ SHO Oral and Maxillo-facial Surgery, Queen Elizabeth Hospital, King's Lynn, Norfolk; ${ }^{2}$ Consultant Oral and Maxillo-facial Surgery, Queen Elizabeth Hospital, King's Lynn, Norfolk; ${ }^{3}$ Consultant Histopathologist, Queen Elizabeth Hospital, King's Lynn, Norfolk

${ }^{*}$ Correspondence to: A. W. Green, 6 King Street, King's Lynn, Norfolk PE30 1ES

REFEREED PAPER

Received 21.08.00; Accepted 15.11.00

(C) British Dental Journal 2001; 190: 529-530
In brief

- Take odontogenic infections seriously

- Importance of regular dental check-ups

- Good oral hygiene and diet habits

- Rapid surgical and antibiotic treatment for spreading facial infections

Two days later the patient reattended. He was pyrexial and had not been taking his antibiotics, as he found it difficult to swallow. Clinically pus was noted escaping from the extra-oral incision. Blood cultures were obtained and found to be positive for Streptococcus intermedius, which was sensitive to penicillin. Intravenous antibiotics were commenced, (benzyl penicillin $600 \mathrm{mg}$ four times a day and metronidazole $500 \mathrm{mg}$ three times a day). Over the next 4 days the
Fig. I Pre-operative orthopantomograph showing carious teeth and periapical pathology extra-oral swelling and swallowing improved and the patient was discharged home, with oral antibiotics and a followup appointment arranged for 1 week later. No further contact was made by the patient. However, on the day of the patient's scheduled follow-up appointment, he was unexpectedly brought to the A\&E department by emergency ambulance, having suffered at home sudden haemorrhaging from the oral cavity and subsequent respiratory arrest. On admission the patient's haemoglobin was $5.8 \times 10^{9} \mathrm{~g} / \mathrm{dl}$. Initial examination revealed that the haemorrhage was arising deep within the aerodigestive tract and not from the mouth.

Despite extensive resuscitation measures the patient suffered an electromechanical dissociation arrest and died 2 hours later.

At the post-mortem performed there, an abscess cavity was found at the root of the neck which communicated with the operation sites. It involved the subclavian vein and partially destroyed it. The subsequent haemorrhage tracked behind the pleura producing a mass and through the pleura into the pleural cavity producing a massive haemothorax measuring $3,750 \mathrm{ml}$.

The chest $\mathrm{x}$-ray on admission showed a mass in the right side of the chest above the carina, which on post-mortem was shown to be blood trapped beneath the parietal

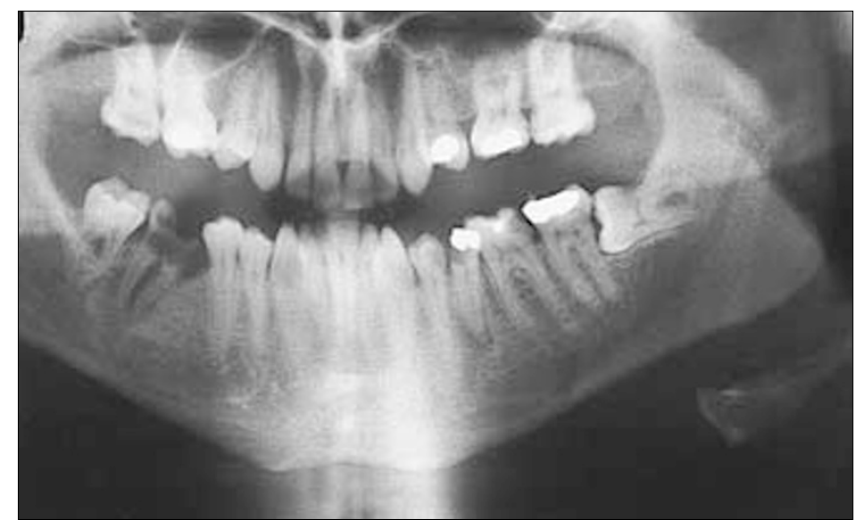


Fig.2 Chest x-ray showing mass in right side of chest above the carina

pleura. The chest $\mathrm{x}$-ray was taken prior to the final bleed (Fig. 2).

\section{Discussion.}

The post-mortem showed the lateral pharyngeal space to be the reservoir of infected material, which had tracked down around the carotid sheath to the root of the neck, where the subsequent haemorrhage occurred. Removal of the original odontogenic cause, supported by appropriate antibiotics, would normally result in resolution of the lateral pharyngeal space infection. ${ }^{4}$ In this particular case, however, infection continued, despite the apparent resolution of the neck swelling.

Further clarification of the situation might have been obtained with additional imaging of the neck. Plain film radiographs (lateral soft tissue C-spine and posterior anterior view of the same region) may have identified infection in the soft tissues. ${ }^{3}$ The ideal imaging modality would be magnetic resonance imaging or ultrasound, which clearly defines the spaces and also helps differentiate cellulitis from an abscess. ${ }^{5}$ Given the clinical picture at the time and the fact that the patient's clinical condition was improving, both in the hospital and at home, there were no indications for further imaging.

In deep neck infections from odontogenic origins the commonest causative organisms are generally Streptococci and oral anaerobes. In this case Streptococcus intermedius was isolated, which was sensitive to penicillin.

Streptococcus intermedius spreads very readily through the soft tissues, because of its ability to produce hyaluronidase and streptokinase, which breaks down ground substance and fibrin. It has been postulated that mixed infections, involving Streptococcus intermedius and Fusobacterium, can produce rapidly spreading infections, because of a symbiotic reaction. ${ }^{6}$ However, Fusobacterium was not cultured in this case.

\section{Conclusion}

This particular case highlights the importance of taking all odontogenic infections seriously and emphasises the need for early treatment and prompt referral when necessary.

1 Zeitoun I M, Dhanarajani P J. Cervical cellulitis and mediastinitis caused by odontogenic infections: report of two cases and review of literature. J Oral Maxillofac Surg 1995; 53: 203-208.

2 Wong T. A nationwide survey of deaths from oral and maxillofacial infections: the Taiwanese experience. J Oral Maxillofac Surg 1999; 57: 1297-1299.

3 Peterson L J. Contemporary management of deep infections of the neck. J Oral Maxillofac Surg 1993; 51: 226-231.

4 Krishnan V, Johnson J V, Helfrick J F. Management of maxillofacial infections. J Oral Maxillofac Surg 1993; 51: 868-873.

5 Fielding A F, Reck S F, Barker W J. Use of magnetic resonance imaging for localization of a maxillofacial infection: Report of a case. $J$ Oral Maxillofac Surg 1987; 45: 548.

6 Peterson L P. Microbiology of head and neck infections. Oral Maxillofac Surg Clin N Am 1991; 3: 247-257. 